\title{
Indian Sign Language Recognition
}

\author{
Ashwitha Anjali Maben*, Prajna Shetty, Mamatha Salian
}

Computer science and Engineering, Srinivas Institute of Technology, Mangalore, Karnataka, India

\section{ABSTRACT}

\section{Article Info}

Volume 7, Issue 4

Page Number: 149-152

Publication Issue :

July-August-2021

\section{Article History}

Accepted : 06 July 2021

Published : 13 July 2021
The paper focuses on designing and developing an user interface to help out the dumb community in making a better use of their gestures. It mainly deals with making services of the hand gestures to be easily accessible and understandable to by the people using sign languages. This system makes use of data collected from standards of Indian Sign Languages (ISL). The system is built using machine learning tools, TensorFlow library. The result of this project is to translate the gestures into the texts and return it to the web interface. The sole reason of this project is to help the individual with speech and hearing impairment.

Keywords: Indian Sign Language, Tensorflow, Extraction Algorithm

\section{INTRODUCTION}

Mute is a disability that impair their speaking and deaf is a disability that impair their hearing. The difficult thing to them to communicate with normal people. Not all the people know the sign languages it makes communication between impaired people and other difficult. What if there is away for normal people and deaf-mute people to communicate. As we know the only way for them to communicate is through sign language and the normal people tend to ignore the importance of sign languages. While sign language takes, place a very important role in deafmute people, to communicate both with normal people and with themselves. There is deaf Interpreter who provides translation services. However, these Interpreters are high-priced. Hence, there is a requirement of inexpensive solution.
The whole idea is to build a communication system that enables communication between normal person and speech hearing impaired. There have been several research has been done to help the people who are deaf and dumb. The main use of sign language recognition system is to convert sign language into text so that normal people can understand. Thus, we provide a technique where hand gestures are converted to text so that normal people can understand which will help dumb-deaf people to conveying their views to others.

\section{LITERATURE SURVEY}

Communication is the important aspect of people. Humans act one another to convey their messages, thoughts, ideas and knowledge to the people. However, there are few deaf mute folks around the world. During this paper, idea planned is good glove, which converts linguistic communication to speech

Copyright: @ the author(s), publisher and licensee Technoscience Academy. This is an open-access article distributed under the terms of the Creative Commons Attribution Non-Commercial License, which permits unrestricted non-commercial use, distribution, and reproduction in any medium, provided the original work is properly cited 
outputs. communication for speech impaired person is produced because the glove facilitates in manufacturing artificial speech.This paper shows flex device primarily based gesture recognition module which is developed to acknowledge English alphabet, words and text to speech synthesizer.[2] In this, the paper presents a gesture to speech device for dumb folks. The sign language of dumb is somewhat troublesome to communicate and it is unacceptable for everyone to communicate in that language. Therefore, many people cannot return and share their thoughts with these speech-impaired folks. In this system, a digital camera is placed ahead of the impaired person. The person would place his finger ahead of the net camera and also the digital camera. It captures the hand gesture and performs image processing exploitation principle part analysis algorithmic program (PCA).[3] We humans can mainly be able to make a point or communicate with others through sound and vision. Hand gesture also helps in identifying motions through expressions. Thoughts, messages and ideas can be exchanged with hand gestures very well. Using the concept of Thresholding numbers from 0 to 10 are recognized using the algorithm. Basically there are three steps: capturing the image, applying threshold and then recognizing the numbers. To complete the above process, user must be aware of the use of colour hand gloves.[4] Expressions as well as hand motions are used by the speech impaired person to communicate with others. Artificial mouth for speaking, a new technique called is proposed here. This system is based on monitoring sensors, It helps the impaired person to convey their thoughts. Motion helps in easy understanding but not every time, as various information or messages needs to be conveyed. Using the dumb communication interpreter hand gestures are converted to sensibility speech.[5] These days linguistic communication recognition is one amongst the foremost growing fields of analysis. A symbol language consists of varied gestures fashioned by physical movement of body components, a technique is planned that produces the employment of hand gestures for recognition of Indian linguistic communication.[6] The project mainly focuses on reducing the communication gap between the physically impaired person and the normal ones. Here, hand gloves are fitted with flex sensors which helps in interpreting numbers and English letters in American Sign Language (ASL) and some in the Indian standard one.[7] This paper is planned in such a way as to assist these specially challenged individuals hold equal par in the society.

\section{IMPLEMENTATION}

The system implementation includes several steps in which loading of user interface that is the web user interface is done at the beginning. In this all, the main class function which require permission for webcams and training modules is loaded. In the next step, different ways of representing gesture, which will be stored in datasets, are trained. When all the gestures are trained, using squeeze.net library there are loaded into KNN classifier function. The webcam then has the permission to recognize all the gestures, which will be then predicted by the system.

In this system implementation, the theoretical design will be converted into a working model. The system is implemented using Java script, Node.js, Tensor flow, HTML, Bootstrap and Visual Studio. The design of the project includes giving a user interface to enter hand signs, which will be stored in database. The datasets use algorithms like KNN for clustering different signs. The user is also given an option to either train the model or directly make use of stored dataset. The output of the system is in form of texts. 


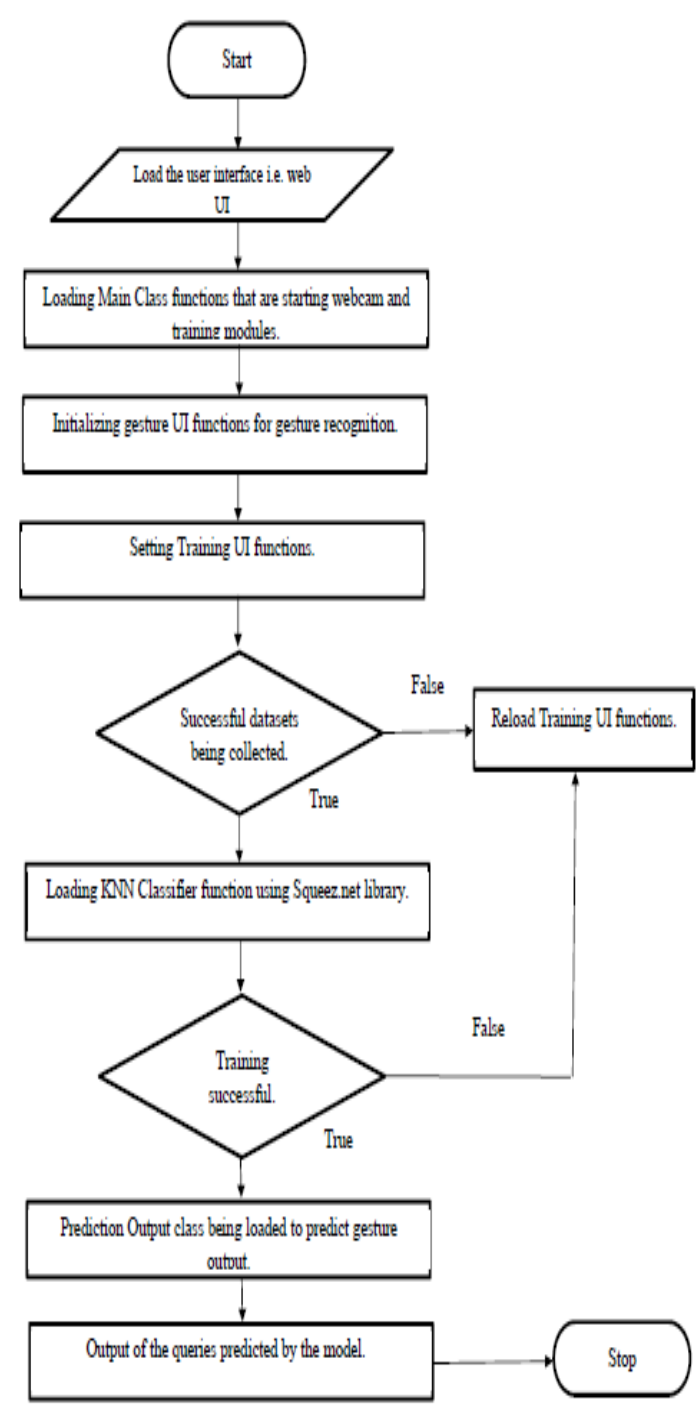

Fig 3.1: System Implementation

\section{RESULTS AND DISCUSSION}

The system is being designed and following outputs have been gained as results. Moreover, the system is in prototype state, the results obtained were promising but to improve overall accuracy, we ensured that all the requirement conditions are met. Figure 4.1 shows the homepage of the application. Where user needs to enter the URL to enter this page. This page contains all information about the web application. User can move forward by clicking training button provided in the top right. The figure 4.2 shows how to train hand gestures in different angles. In order to obtain maximum accuracy atleast minimum of 30 samples of data sampled has to be trained. The figure 4.3 shows Adding guesture to page. The text box will be provided with the option of training the guestures as mentioned above where user has to enter the required guesture name in it by capturing the dataset samples in the web cam.The figure 4.4 is prediction page It shows the model which predicts the start guesture and also one could see that in the a window showing the accuracy level at which the guesture is being predicted

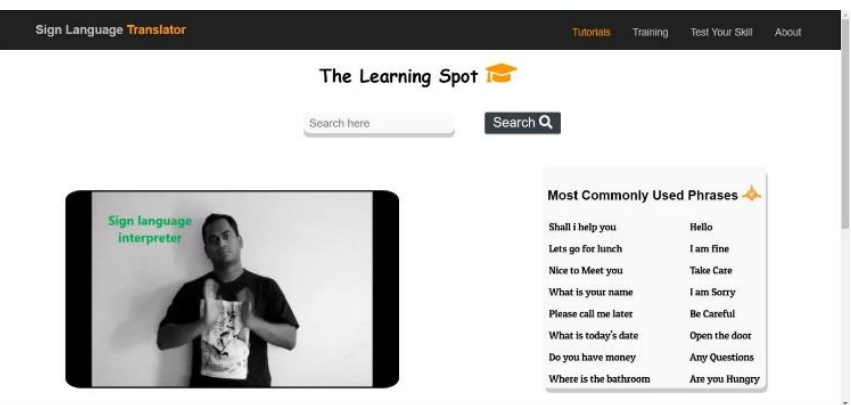

Fig 4.1: Home Page

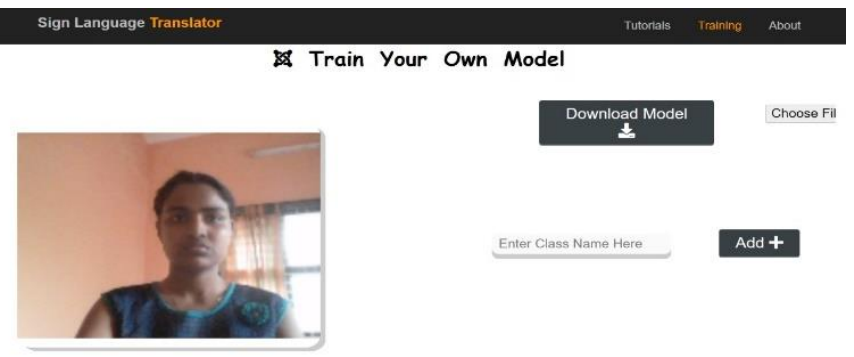

Fig 4.2: Training Initial Gesture Page

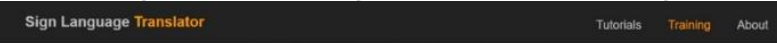

Train Your Own Model
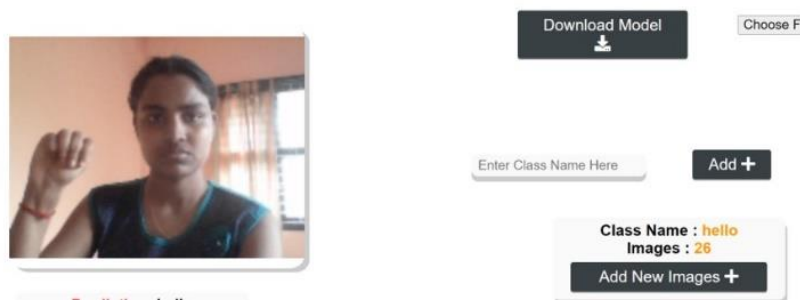

Fig 4.3: Training Gesture Page 

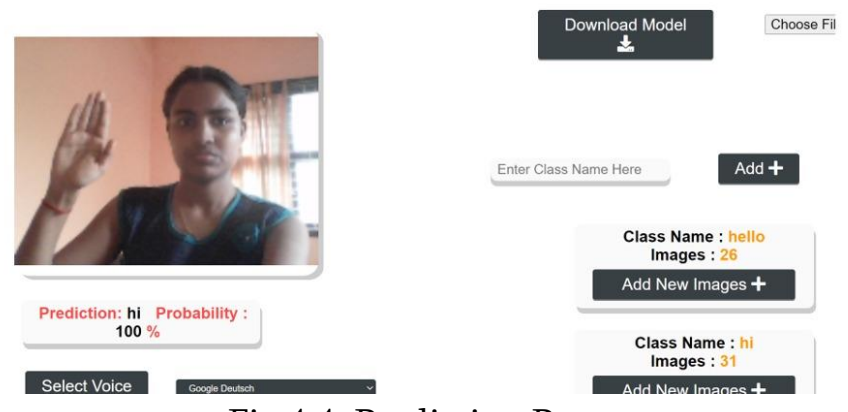

Fig 4.4: Prediction Page

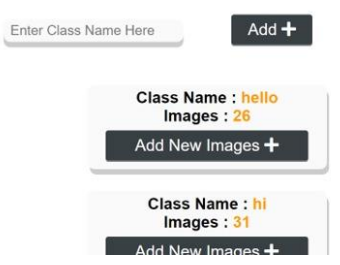

\section{CONCLUSION}

The problem of deaf and dumb candidates, resolved by creating a web application. This web application allows the users to interact with the people of real world with the sign language gestures. It allows the user to train their custom made as well as standard sign language gestures to interact with the normal user to do the day-to-day tasks. It connects the gap between the mutual communication problems caused due to lack of knowledge of sign languages to normal users. This web app results in efficient time usage. The system helps to improve the performance. Maintaining the project is easy and manageable. It is easily understandable by the user. Future work that can be added to this project is, the web application can be converted into android applications. It can be modified in such a way that application can be used by organizations other than educational institutions. Organizations can add functionalities depending on their needs.

\section{REFERENCES}

[1]. Ashish G. Bairagi, Y.D. Kapse P.G. Student, Department of E\&TC Engineering, GCOEJ, Jalgaon, Maharashtra, "Survey on Sign language to Speech Conversion", International Journal of Innovative Research in Computer and Communication Engineering, 2018.

[2]. Abhishek Jain, Lakshita Jain, Ishaan Sharma, Abhishek Chauhan, TECH, Department Of ECE, SRMIST," Image Processing Based Speaking System
For Mute People Using Hand Gestures", International Journal Of Engineering Sciences \& Research Technology, 2018.

[3]. Bhavsar Swapna1, Futane Pravin1 and V. Dharaskar Rajiv, Sinhagad college of engineering, Pune University," Hand Gesture Recognition System for Numbers Using Thresholding", Research Center Amravati University Amravati, India, 2018.

[4]. V.Padmanabhan, M.Sornalatha," Hand gesture recognition and voice conversion system for dumb people", International Journal of Scientific \& Engineering Research, 2014.

[5]. Aruljothy, Arunkumar, Ajitraj, Yayad Damodran, Jeevanantham, Dr.M.Subba, UG Scholar, Department of Instrumentation and Control Engineering, Sri Manakula Vinayagar Engineering College, Puducherry," Hand Gesture Recognition Using Image Processing for Visually Impaired and Dumb Person", International Journal of Advanced Research in Computer and Communication Engineering ISO, 2014.

[6]. Sunita V. Matiwade, DR. M.R.Dixit Student, Guide Department of Electronics and Telecommunication, KIT's College of Engineering, Kolhapur, "Electronic Support System to Interpret Sign Language of Communication used by Deaf and Dumb Community", Journal of Emerging Technologies and Innovative Research, 2015.

[7]. Rupesh Prajapati, Vedant Pandey, Nupur Jamindar, Neeraj Yadav, Prof. Neelam Phadnis," Hand Gesture Recognition and Voice Conversion for Deaf and Dumb", International Research Journal of Engineering and Technology, 2018.

\section{Cite this article as :}

Ashwitha Anjali Maben, Prajna Shetty, Mamatha Salian, "Indian Sign Language Recognition", International Journal of Scientific Research in Computer Science, Engineering and Information Technology (IJSRCSEIT), ISSN : 2456-3307, Volume 7 Issue 4, pp. 149-152, July-August 2021. Available at doi : https://doi.org/10.32628/CSEIT217436

Journal URL : https://ijsrcseit.com/CSEIT217436 